\title{
PATOLOGIAS RELACIONADAS AO SISTEMA NERVOSO CAUSADAS POR TRAUMATISMOS CRANIOENCEFÁLICOS EM CONSEQUÊNCIA DE ACIDENTES AUTOMOBILÍSTICOS
}

Conrodo Lenzi Pereira, João Vitor Ferreira dos Reis, Lorena Pinheiro de Souza, Madalena Henriger Chamon Esteves da Costa, Yasmim Vilela Rodrigues, Moacir Ferreira Júnior. Patologias relacionadas ao sistema nervoso causadas por traumatismos cranioencefálicos em consequência de acidentes automobilísticos. Revista Saúde Dinâmica, vol. 2, núm.2, 2020. Faculdade Dinâmica do Vale do Piranga. 


\title{
Patologias relacionadas ao sistema nervoso causadas por traumatismos cranioencefálicos em consequência de acidentes automobilísticos
}

\author{
Pathologies related to the nervous system caused by cranioencephalic trauma as a result of automobile \\ acidentes
}

Conrado Lenzi Pereira ${ }^{l}$, João Vitor Ferreira dos Reis ${ }^{l}$, Lorena Pinheiro de Souza ${ }^{1}$, Madalena Heringer Chamon Esteves da Costa ${ }^{l}$, Yasmim Vilela Rodrigues ${ }^{1}$, Moacir Ferreira Júnior ${ }^{2}$

${ }^{I}$ Discentes do Curso de Medicina da Faculdade Dinâmica do Vale do Piranga - FADIP, Rua G, lote 11, 205

- Bairro Paraíso - Ponte Nova-MG CEP: 35.430-302.

${ }^{2}$ Docente do Curso de Medicina da Faculdade Dinâmica do Vale do Piranga - FADIP, Rua G, lote 11, 205 - Bairro Paraíso - Ponte Nova - MG CEP: 35.430-302.

Autor correspondente: lenziconrado@gmail.com

\section{Resumo}

Devido a grande prevalência de acidentes automobilísticos na contemporaneidade é preciso elucidar os seus mecanismos de ocorrência bem como suas possíveis consequências ao TCE e às patologias do sistema nervoso, possibilitando a construção de medidas que minimizem potenciais danos, como a epilepsia e os danos cerebrais potencialmente, fatais. O objetivo deste artigo é esclarecer quais são as kpatologias que podem ser desencadeadas diante de tais episódios, bem como o impacto delas na sociedade. $\mathrm{O}$ estudo configura-se como uma revisão integrativa realizada nas bases de dados LILACS, MEDLINE, SCIELO, no Portal do DER-MG, tendo como metodologia a pesquisa retrospectiva quantitativa e qualitativa. Esta revisão demonstrou uma grande prevalência de patologias relacionadas ao sistema nervoso causadas por traumatismos cranioencefálicos oriundos de acidentes e traumas automobilísticos, os quais configuram um grande problema de saúde pública, sobrecarregando os hospitais e emergências subordinadas ao SUS.

Palavras-chave: traumatismos cranioencefálicos, sistema nervoso, neurocirurgia, patologia, epidemiologia

\begin{abstract}
Due to the high prevalence of automobile accidents in contemporany times, it is necessary to elucidate their mechanisms of occurrence as well as their possible consequences to TCE and nervous system pathologies, enabling the construction of measures that minimize potential damage, such as epilepsy and brain damage potentially fatal. The objective of this article is to clarify what are the pathologies that can be triggered in the face of such episodes, as well as their impact on society. The study is configured as an integrative review carried out in the LILACS, MEDLINE, SCIELO databases, on DER-MG Portal, using the methodology of quantitative and qualitative retrospective research. This review demonstrated a high prevalence of pathologies related to the nervous system caused by traumatic brain injuries resulting from car accidentand traumas, which constitute a major public health problem, overburdening hospitals and emergencies subordinated to SUS.

Keywords: traumatic brain injuries, nervous system, neurosurgery, pathology, epidemiology
\end{abstract}




\section{INTRODUÇÃO}

O Traumatismo Cranioencefálico (TCE), segundo de Albuquerque et al. (2016), é definido como qualquer lesão decorrente de um trauma externo, que tenha como consequência alterações anatômicas do crânio, como fratura ou lacerações do couro cabeludo, bem como o comprometimento funcional das meninges, encéfalo ou seus vasos, resultando em alterações cerebrais, momentâneas ou permanentes, de natureza cognitiva ou funcional.

A lesão encefálica definitiva que se estabelece após o TCE, de acordo com Fukujima (2013), é o resultado de mecanismos fisiopatológicos que se iniciam com o acidente e se estendem por dias a semanas. Assim, do ponto de vista didático, as lesões cerebrais são classificadas em primárias e secundárias. As lesões primárias são aquelas que ocorrem no momento do trauma enquanto que as lesões secundárias decorrem de agressões que se iniciam após o acidente, resultantes da interação de fatores intra e extracerebrais.

Os acidentes de trânsito constituem, atualmente, um importante problema de saúde pública no Brasil e no mundo, sendo considerados relevantes fatores de morbimortalidade. Estudos apontam diversos fatores associativos ä ocorrência desses sinistros, dentre os quais a má sinalização das estradas, a pouca iluminação das vias, a inexperiência do condutor, a direção em alta velocidade, a ingestão de bebidas alcoólicas, a imprudência no trânsito e a utilização do aparelho celular ao dirigir (MARINHO et al., 2019).

De Souza, Regis e Koizumi (1999) afirmam que a taxa de óbito de pedestres e motociclistas, vítimas de acidentes de trânsito, é mais elevada quando comparada a outras causas, entre as quais: neoplasias e doenças cardiovasculares. Os motociclistas, entretanto, são indivíduos que frequentemente sofrem lesões graves em múltiplos sistemas resultando em consequências devido à ausência de proteção.

As estatísticas indicam que a maior parte das vítimas dos acidentes automobilísticos é do sexo masculino e alocadas nas faixas etária de 1 a 44 anos. Além disso, os números indicam que esses acidentes são causas importantes de letalidade, incapacidade e mortalidade para esta parcela da população. O sexo masculino é o mais acometido. Sendo assim, as lesões traumáticas decorrentes dos acidentes automobilísticos correspondem a cerca de $10 \%$ do total de mortes por causas externas. Os Traumatismos cranioencefálicos (TCEs) ainda são responsáveis por 75\% a 
97\% das mortes por trauma em crianças (DE ALBUQUERQUE et al., 2016; RUY; DA ROSA, 2011).

De acordo com Ascari et al. (2013), pacientes acometidos com TCE apresentam risco duzentas vezes maior, que a população geral, de desenvolver epilepsia, tornando-se uma causa relevante do desenvolvimento da epilepsia secundária, assim como infecções e uso abusivo de drogas. Além desta patologia, o TCE é também uma das principais causas de incapacidade motora e cognitiva.

Diante ao exposto e devido a grande prevalência de acidentes automobilísticos na contemporaneidade é preciso elucidar os seus mecanismos de ocorrência bem como suas possíveis consequências, proporcionando meios que possam minimizar desdobramentos que comprometem a qualidade de vida do indivíduo como: a epilepsia e a hipertensão intracraniana.

Com isso, o objetivo deste artigo é esclarecer quais são as patologias que podem ser desencadeadas diante de tais episódios.

\section{MATERIAIS E MÉTODOS}

A referida revisão integrativa de literatura foi realizada a partir das bases de dados online SCIELO (Scientific Eletronic Library Online), LILACS (Literatura Lantino-Americana do Caribe em Ciências da Saúde) acessadas através da Biblioteca Virtual em Saúde (BVS). Além disso, a busca também ocorreu na base de dados MEDLINE, no Portal do Departamento de Estradas e Rodagens de Minas Gerais - DER-MG, no Portal do Departamento Nacional de Trânsito - DENATRAN e no Portal do Corpo de Bombeiros de Minas Gerais - CBM/MG. No processo de busca foram utilizados os seguintes descritores: [traumatic brain injuries], [nervous system], [neurosurgery], [pathology] e [epidemiology]. Para que o processo de busca se tornasse mais efetivo foram utilizados os filtros: text availability (full text), language (portuguese and english) e article type (review). A busca ocorreu durante o mês de setembro de 2019 e foram selecionados artigos que preenchia os seguintes critérios: ser um estudo retrospectivo quantitativo e qualitativo, epidemiológico com abordagem da ocorrência do trauma crânio-encefálico, da sua etiologia e de suas consequências patológicas bem como os artigos que traçavam o panorama de incidência de tais traumas e patologias no Brasil. Foram excluídas duplicidades. Um total de 22 pesquisas foi selecionado atendendo a esses critérios. 


\section{RESULTADOS E DISCUSSÃO}

Fukujima (2013) afirma que o traumatismo crânio-encefálico (TCE) é o principal determinante de morbidade, incapacidade e mortalidade dentro deste grupo. O TCE grave está associado a uma taxa de mortalidade de $30 \%$ a $70 \%$, e a recuperação dos sobreviventes é marcada por sequelas neurológicas graves e por uma qualidade de vida muito prejudicada. No conjunto de lesões das causas externas, o TCE destaca-se em termos de magnitude e, sobretudo, como causa de morte e incapacidade. As lesões cerebrais e suas coberturas ocorrem em aproximadamente 200 por 100.000 pessoas por ano e respondem por 14 a 30 mortes por 100.000 pessoas por ano, nos Estados Unidos. Os homens são acometidos duas ou três vezes mais frequentemente que as mulheres. A gravidade do problema é complicada pelas sequelas comportamentais até mesmo em função das lesões cranianas relativamente pequenas. A baixa idade das vítimas magnifica as perdas pessoais e sociais relacionadas à lesão craniana.

Os coeficientes encontrados são 2,5/100 mil (3,6/100 mil para os homens e 1,5/100 mil para as mulheres). Mostraram ainda que foi um dos principais e frequentes motivos de hospitalização correspondendo a 9,9\% das internações, logo após fraturas com 42,6\% e causas como neoplasia, doenças cardiovasculares, diabetes doenças respiratórias entre outras estando com $14,2 \%$ das internações no ano de 2000. Ademais, elucida as principais causas de TCE que levam a internação e se observa que as quedas são responsáveis por $40 \%$ das causas de internação, seguidas dos acidentes de transporte $(32,4 \%)$ e demais causas $(19,2 \%)$ (GAUDÊNCIO; LEÃO, 2013).

Corroborando com isso, Ascari et al. (2013) relata ter verificado que os tipos de traumatismos mais frequentes foram as escoriações (43\% dos casos), contusões (24\% dos casos), fraturas (22\% dos casos), seguidos de lesões corto- contusas (10\%). Considerando que o meio de transporte mais utilizado pelas vítimas de acidentes de trânsito foi à motocicleta, as lesões encontradas na população pesquisada são justificadas. Nos acidentes automobilísticos, a parte do corpo mais atingida são,os membros superiores e inferiores (79\% dos casos), crânio ( $39 \%$ dos casos), tórax ( $7 \%$ dos casos), abdome (3\% dos casos) e a pelve ( $2 \%$ dos casos). Nos membros supracitados, o tipo mais frequente de lesões são as escoriações, somando $26 \%$ dos casos, seguido das contusões, com $12 \%$ dos casos. Os traumas no crânio somaram $30 \%$ dos 
casos, sendo os mais relevantes neste tipo de atendimento. Nos acidentes com moto, os membros, superiores e inferiores, foram o segmento anatômico mais atingido, somando 92\% dos casos atendidos, e as principais lesões encontradas foram escoriações (53\% dos casos), fraturas (20\% dos casos), contusões (11\% dos casos) e ferimentos corto contusos (8\% dos casos).

Em Barbalha 79,3\% das vítimas não usavam capacete no momento do acidente, assim como 60,21\% da amostra do estudo realizado em Teresina. Na pesquisa realizada em São José do Rio Preto, esse dado não foi relatado. Em síntese, nos referidos estudos percebeu-se que a maioria dos tipos de acidente causador de TCE foi a colisão, estando relacionados, principalmente ao sexo masculino e com a ausência de mecanismos e dispositivos de proteção (GAUDÊNCIO; LEÃO, 2013).

No TCE, a presença de elementos como hematomas, contusões, edema, acúmulo de líquido cefalorraquidiano (LCR) ou aumento do volume intravascular, que superar a capacidade de acomodação intracraniana, elevam a PIC (pressão intracraniana). A HIC (hipertensão intracraniana) foi encontrada em $71 \%$ dos pacientes operados com hematoma intracerebral e $39 \%$ dos pacientes com HSDA e HED (DE ANDRADE et al., 2009).

Dentre as causas da HIC, a que confere os maiores desafios é o edema, que tem fisiopatologia e manuseio complexos. Existem cinco tipos principais de edema: citotóxico, vasogêncio, hidrostático, intersticial e osmótico.

O edema citotóxico tem como causa a ausência do aporte de energia, que interfere com os mecanismos da bomba iônica na membrana celular e leva ao acumulo de sódio intracelular e consequente edema.

O edema cerebral vasogênico decorre de distúrbio na barreira hemato encefálica permitindo a passagem de água, sódio e proteína para dentro do espaço intersticial. O edema é formado na substância cinzenta, mas pode acumular-se na substância branca.

O edema hidrostático ocorre devido ao acúmulo de líquido extracelular que, apesar de, pobre em proteína promove um aumento abrupto na diferença de pressão hidrostática entre os espaços intra e extravascular.

O edema intersticial é uma complicação da obstrução do LCR que ocorre na hidrocefalia. O aumento da pressão intraventricular força a infiltração de LCR no tecido periventricular. 
De acordo com de Andrade et al. (2009); Gaudêncio e Leão (2013), o edema osmótico ocorre devido a diminuição da osmolaridade plasmática usualmente associado à hiponatremia.

O impacto dessas sequelas é intenso, sendo um fator que dificulta o retorno para as atividades diárias. Após um TCE de grande impacto, seguido por contusões cerebrais ou mesmo ferimentos penetrantes, o risco para o desenvolvimento de crises epilépticas aumenta consideravelmente, sendo necessários estudos que mostrem a eficácia das estratégias profiláticas às crises. A probabilidade de ocorrência da crise epiléptica é diretamente proporcional à gravidade do TCE. Estudos revelam que após TCE, o risco global para o desenvolvimento de crises epilépticas fica em torno de $2 \%$ a $5 \%$, aumentando para $7 \%$ a $39 \%$ naqueles com lesões corticais ou sequelas neurológicas. O primeiro ano após o TCE é o período crítico de maior probabilidade para uma crise epiléptica, diminuindo nos anos subsequentes (DE ANDRADE et al., 2009).

D’Ambrósio e Perucca (2004) descrevem que os fatores de risco independentes para a doença incluem a presença de um hematoma subdural ou mesmo extradura e contusão cerebral. Fatores de risco independentes considerados adicionais incluem: período de coma maior que uma semana; lesão dural; fraturas de crânio depressivas sem tratamento cirúrgico.

Por fim, as incapacidades resultantes do TCE podem ser divididas em 3 categorias: físicas, cognitivas e emocionais/ comportamentais. As físicas são diversificadas, podendo ser motoras, visuais e táteis. As cognitivas, frequentemente, incluem problemas de atenção, memória e funções executivas. As incapacidades comportamentais e emocionais são, em geral, a perda de autoconfiança, motivação diminuída, depressão, ansiedade, dificuldade de autocontrole; está representada mais comumente por desinibição, irritabilidade e agressão.

\section{CONCLUSÃO}

O referido estudo demonstrou grande prevalência de patologias relacionadas ao sistema nervoso, como a epilepsia e a hipertensão intracraniana, causadas, em suma, por traumatismos cranioencefálicos em consequência dos acidentes automobilísticos e também suas consequências como incapacidades motoras, fraturas, contusões, escoriações, as quais limitam amplamente a qualidade de vida do indivíduo, requerendo uma maior política de atenção, principalmente no que tange ao planejamento de ações preventivas e de conscientização. 


\section{REFERÊNCIAS}

ASCARI, Rosana Amora; CHAPIESKI, Cristiane Maria; DA SILVA, Olvani Martins; FRIGO, Jucimar. Perfil Epidemiológico de Vítimas de Acidentes de Trânsito. Rev Enferm UFSM, [s. l.], v. 3, ed. 1, p. 112-121, 2013.

D'AMBRÓSIO, Raimondo; PERUCCA, Emilio. Epilepsy after head injury. Curr Opin Neurol, [s. l.], v. 17, ed. 6, p. 731-735, dez. 2004.

DE ALBUQUERQUE, Adriana Montenegro; SILVA, Helder Clayton de Lima; TORQUATO, Isolda Maria Barros; GOUVEIA, Bernadete de Lurdes André; ABRANTES, Magaly Suênya de Almeida Pinto; TORRES, Valdicléia da silva Ferreira. VÍTIMAS DE ACIDENTES DE MOTO COM TRAUMATISMO. Rev enferm UFPE , Recife, v. 10, ed. 5, p. 1070-1078, maio 2016.

DE ANDRADE, Almir Ferreira; PAIVA, Wellingson Silva; DE AMORIM, Robson Luis Oliveira; FIGUEIREDO, Eberval Gadelha; NETO, Eloy Rusafa; TEIXEIRA, Manoel Jacobsen. Mecanismos de Lesão Cerebral no Traumatismo Cranioencefálico. Rev Assoc Med Bras , [s. l.], v. 55, ed. 1, p. 75-81, mar. 2009.

DE SOUZA, Regina M C; REGIS, Fabiane C; KOIZUMI, Maria S. Traumatismo crânioencefálico: diferenças das vítimas pedestres e ocupantes de veículos a motor. Rev. Saúde Pública, [s. l.], v. 33, ed. 1, p. 85-94, fev. 1999.

FUKUJIMA, Marcia Maiumi. O Traumatismo Cranioencefálico na Vida do Brasileiro. Rev Neurocienc, [s. l.], v. 21, ed. 2, p. 173-174, 2013.

GAUDÊNCIO, Talita Guerra; LEÃO, Gustavo de Moura. A Epidemiologia do Traumatismo CrânioEncefálico: Um Levantamento Bibliográfico no Brasil. Rev Neurocienc, [s. l.], v. 21, ed. 3, p. 427-434, 2013.

MARINHO, Cristiane da Silva Ramos et al. Acidente de trânsito: análise dos casos de traumatismo cranioencefálico. Enfermería Global , [s. l.], ed. 54, p. 333-342, abr. 2019.

RUY, Érica Lopes; DA ROSA, Maria Inês. Perfil epidemiológico de pacientes com traumatismo crânio encefálico. Arquivos Catarinenses de Medicina, [s. l.], v. 40, ed. 3, p. 17-20, 2011.

\section{Declaração de Interesse}

Os autores declaram não haver nenhum conflito de interesse

\section{Financiamento}

Financiamento próprio

\section{Agradecimentos}


Agradecemos o Prof Dr. Moacir Ferreira Júnior e a Prof ${ }^{a}$ Dra. Bruna Soares de Souza Lima Rodrigues pela orientação e atenção na elaboração deste artigo.

\section{Colaboração entre autores}

Conrado Lenzi Pereira: pesquisa e levantamento bibliográfico, redação, elaboração e submissão do artigo.

João Vitor Ferreira dos Reis: pesquisa e levantamento bibliográfico, redação do artigo. Lorena Pinheiro de Souza: pesquisa e levantamento bibliográfico.

Madalena Heringer Chamon Esteves da Costa: pesquisa e levantamento bibliográfico. Yasmim Vilela Rodrigues: pesquisa e levantamento bibliográfico, redação do artigo.

Moacir Ferreira Júnior: orientação e supervisão do artigo. 